\title{
The Operational and Financial Value of an Interventional Radiology Clinic at a Large, Academic, Tertiary Public Hospital System
}

\author{
Joseph L. McDevitt ${ }^{1} \quad$ Rehan S. Quadri $\quad$ Patrick D. Sutphin ${ }^{1} \quad$ Mark Reddick ${ }^{1}$ \\ ${ }^{1}$ Department of Radiology, University of Texas-Southwestern \\ Medical Center Dallas, Texas, United States

\begin{abstract}
Address for correspondence Joseph L. McDevitt, MD, MBA, Department of Radiology, UT Southwestern Medical Center, 5323 Harry Hines Blvd, Dallas, TX 75390, United States (e-mail: josephlmcdevitt@icloud.com).
\end{abstract}

J Clin Interv Radiol ISVIR:2021;5:16-21

\begin{abstract}
Purpose To evaluate the operational and financial impact of an interventional radiology (IR) clinic at a tertiary county hospital system.

Methods The IR clinic, which opened in January 2017, evaluates outpatient referrals and completes preprocedure workups, nonimage-guided procedures, and postprocedure follow-up visits. Procedural volumes, locations, start times, and end times were analyzed from September 2015 to June 2018, with comparison of pre- and postclinic values by $t$-tests.

Results Relative to the preclinic period, the number of IR cases completed each quarter has increased by an average of $12 \%$ (pre: $953 \pm 63$, post: $1063 \pm 34, p=0.01$ ). Procedures that saw the largest quarterly growth included port placements (44\% increase; pre: $82 \pm 8$, post: $118 \pm 17, p=0.002$ ), inferior vena cava (IVC) filter placements (24\% increase; pre: $33 \pm 12$, post: $41 \pm 8, p=0.20$ ), IVC filter removals (72\% increase; pre: $18 \pm 7$, post: $31 \pm 8, p=0.02$ ), and treatments of lower extremity venous disease ( $100 \%$ increase; pre: $7 \pm 2$, post: $14 \pm 6, p=0.04)$. Completion of

Keywords

- interventional radiology

- clinic-based model

- public hospital systems

- quality improvement 119 cases/quarter in clinic (removal of tunneled catheters and infusion ports), as well as a reduction of emergent nephrostomy exchanges, reduced quarterly facility charges for these procedures by $\$ 350,000$. Since the opening of the IR clinic, the first outpatient case started 36 minutes earlier $(p<0.001)$ and the last case finished 19 minutes earlier $(p=0.004)$.

Conclusion Opening an IR clinic resulted in a significant increase in case volume while reducing avoidable costs and improving efficiency of the angiography suite.
\end{abstract}

\section{Introduction}

The interventional radiology (IR) clinical model, with an increased emphasis on inpatient consults and longitudinal outpatient evaluation, is a key component of the Imaging 3.0 Initiative of the American College of Radiology. ${ }^{1-4}$ Specifically, the clinical model has included the development of both dedicated clinical service lines ${ }^{5}$ and outpatient

published online January 25, 2021
DOI https://doi.org/ $10.1055 / \mathrm{s}-0041-1723045$ ISSN 2457-0214. clinics. ${ }^{6}$ Expansion of clinic-based resources has several potential advantages, including improved perceptions of quality of care and understanding of medical conditions, ${ }^{7}$ improved monitoring of chronic vascular conditions, ${ }^{8}$ improved patient safety, ${ }^{9}$ growth in procedural volumes ${ }^{5,10}$ and lower costs, particularly if some procedures can be completed in a clinic-based setting. ${ }^{1}$ Additionally, if preprocedural history and physicals and laboratory evaluations are

(c) 2021. Indian Society of Vascular and Interventional Radiology. This is an open access article published by Thieme under the terms of the Creative Commons Attribution-NonDerivative-NonCommercial-License, permitting copying and reproduction so long as the original work is given appropriate credit. Contents may not be used for commercial purposes, or adapted, remixed, transformed or built upon. (https://creativecommons.org/licenses/by-nc-nd/4.0/).

Thieme Medical and Scientific Publishers Pvt. Ltd. A-12, 2nd Floor, Sector 2, Noida-201301 UP, India 
completed in clinic prior to the day of the procedure, there is an opportunity to improve work hours and angiography suite efficiency.

However, several barriers to more widespread use of clinics exist, including cost of clinic space and opportunity cost for clinical staff. At the institution of this study, a tertiary county hospital, a clinic was introduced to address the problems of insufficient procedural space and inability to workup and manage outpatients. To assess the value of the clinic, the operational and financial benefits to the health system were analyzed by comparing procedure growth, avoided costs, and measures of angiography suite efficiency in the first 18 months after the hospital system opened an outpatient IR clinic.

\section{Methods}

\section{Inclusion Criteria}

This quality improvement project was exempted from review by the university institutional review board. All IR procedures and clinic visits from September 2015 to June 2018 were included for analysis. September 2015 was chosen as the start date because this hospital system opened a new acute care hospital in August 2015, with additional angiography suites relative to the previous acute care hospital, so prior operational data would have provided a misleading comparison set. Notably, in this hospital system, paracentesis and superficial and deep biopsies (with the exception of lung biopsies) are completed by the abdominal imaging division outside of the angiography suite and were not included in this review.

\section{Description of IR Clinic}

The IR division at this 850-bed county hospital system is supported by 3.5 clinical attendings each day, and complete procedures in four angiography suites and have part-time use of a CT room for CT-guided procedures, completing approximately 4,000 cases each year. Efficient resource utilization and reduction in avoidable care is a priority for the hospital and the division, as $50 \%$ of patients are uninsured, $35 \%$ are on Medicaid, and $15 \%$ have private insurance or Medicare. Additionally, 50\% of patients have limited English proficiency.

The IR clinic opened in January 2017 and has a dedicated clinical space in an outpatient building connected to the acute care hospital. It is staffed by a full-time physician assistant, full-time nurse navigator, and clinic scheduler who are solely dedicated to the clinic, and half-time by an attending physician, who also reads vascular imaging during their clinic half-days. ${ }^{11,12}$ All of the clinic staff represented incremental hires to cover this additional clinical work and are costs that are allocated to the clinic. The clinic has a procedure room but does not have a C-arm. The clinic does not have a dedicated ultrasound machine but is located adjacent to the outpatient ultrasound department, so a machine can be used as needed. Formal ultrasound studies are completed in the adjacent outpatient department.
The clinic evaluates outpatient referrals, preprocedure workups and postprocedure follow-up visits and completes nonimage-guided procedures under local anesthesia. All patients who are to receive outpatient procedures in the angiography suite complete a preprocedural visit approximately 1 week prior to the procedure in which the history and physical, informed consent, and preprocedural laboratory analysis are completed. Hepatocellular carcinoma (HCC) patients underwent the same evaluation but in the multidisciplinary HCC clinic. Patients referred for outpatient workup receive a full history and physical examination and receive orders for imaging or other ancillary studies. Additionally, patients with long-term nephrostomies visit the clinic once between each scheduled exchange in the angiography suite for ongoing education (after initial education on the day of the procedure), nephrostomy evaluation (evaluation of the entry site and security of the catheter), and troubleshooting. Postprocedural patients are otherwise seen at the discretion of the attending physicians. Prior to the opening of the clinic, patients did not undergo any preprocedural workup prior to the day of the procedure.

\section{Data Sample}

From September 2015 to June 2018, for all cases that were completed in the inpatient angiography suites, the following data were queried from the imaging record system: date of procedure, start time, end time, inpatient/outpatient status, and procedure name. To obtain clinic volume data, a list of all completed appointments was queried and included: date of appointment and reason for visit. Notably, there were no changes in the number of angiography suites, faculty, number of trainees, or hospital certifications during the study period.

\section{Measuring Impact on Patients with Indwelling Nephrostomies}

The IR clinic provided an opportunity to proactively care for patients with indwelling nephrostomy catheters. Prior to opening the clinic, the department had high rates of emergent exchanges as patients lacked the necessary supplies to care for their nephrostomies at home and were often nonadherent to exchange appointments. These emergent exchanges have high patient morbidity, are costly, and have deleterious effects on routinely scheduled cases..$^{13}$ After the clinic opened, patients had follow-up appointments in the clinic 6 weeks after each scheduled nephrostomy exchange, where they received teaching on how to care for their nephrostomy, and providers ensured that they had sufficient supplies. Patients still received nephrostomy exchange procedures in the angiography suite every 3 months.

To estimate the financial impact of this use of the clinic, the expected and observed proportion of emergent exchanges were compared after the opening of the clinic. The expected distributed assumed that the proportion of nephrostomy exchange completed for emergent indications remained unchanged after opening the clinic. The facility fees due to emergent nephrostomy exchanges were estimated from prior work in the same patient population, which showed 
that emergent exchanges (which are a mix of ER encounters, ward admissions, and ICU admissions) have average excess charges of $\$ 29,500$ relative to routine outpatient exchanges. ${ }^{13}$

\section{Clinic Gross Economics}

Clinic gross margin was reported from institutional data, which included the following: gross revenue (facility and professional) and variable costs (staff and variable facility).

\section{Statistical Analysis}

All statistical analysis was completed in Microsoft Excel. Procedures were grouped based on order name (see - Table 1 for complete list of procedures). Quarterly volumes were plotted pre- and postclinic and the difference in means was assessed by two-sample $t$-test, with $p<0.05$ denoting significance. Start and end times of each procedure were recorded and analyzed for differences pre- and postclinic by two-sample $t$-test, with $p<0.05$ denoting significance. Total number of completed clinic visits per month was calculated. Based on the reason for clinic visit, each appointment was classified as one of the following: nonprocedural clinic visit, implantable infusion port removal, tunneled central line removal, gastrostomy removal or exchange.
To calculate the impact of the clinic on facility fees for procedures that were moved from the angiography suite to the clinic, charges per procedure were obtained and are presented in - Table 2 . The hospital is not able to provide costs at the level of the individual hospital encounter. The aggregate charge reduction from moving procedures to the clinic from the angiography suite was calculated based on the mean difference between angiography suite and clinic charges for that procedure and the number of cases moved to the clinic. Professional fees are billed separately but were equivalent between the clinic and angiography suite, and average collections varied between $\$ 75$ and $\$ 225$, depending on the patients' insurance.

\section{Results}

\section{Impact on Overall Clinic Volumes (All Numbers are Quarterly)}

In the first 18 months after the clinic opened, the number of cases completed by the IR service at this hospital system increased by $11.6 \%$ (preclinic: $953 \pm 63$, postclinic: $1063 \pm 34, p=0.01$ ) (-Fig. 1). The increased number of

Table 1 Procedure volumes

\begin{tabular}{|c|c|c|c|c|c|c|c|}
\hline \multirow{2}{*}{$\begin{array}{l}\text { Procedure } \\
\text { Implantable infusion port placement }\end{array}$} & \multicolumn{2}{|c|}{ Preclinic } & \multicolumn{2}{|c|}{ Postclinic } & \multicolumn{2}{|c|}{ Difference } & \multirow{2}{*}{$\begin{array}{l}p \text {-Value } \\
0.002\end{array}$} \\
\hline & 82 & \pm 8 & 118 & \pm 17 & 36 & $44 \%$ & \\
\hline Tunneled dialysis catheter placement & 110 & \pm 6 & 98 & \pm 19 & -12 & $-11 \%$ & 0.200 \\
\hline Nephrostomy exchange & 69 & \pm 6 & 81 & \pm 7.9 & 12 & $17 \%$ & 0.030 \\
\hline Fistulogram & 66 & \pm 12 & 68 & \pm 16 & 2 & $3 \%$ & 0.900 \\
\hline Implantable infusion port removal & 55 & \pm 5 & 58 & \pm 10 & 3 & $5 \%$ & 0.487 \\
\hline Percutaneous biliary procedures & 54 & \pm 14 & 52 & \pm 8 & -2 & $-4 \%$ & 0.793 \\
\hline Nephrostomy placement & 45 & \pm 6 & 47 & \pm 12 & 2 & $4 \%$ & 0.781 \\
\hline Tunneled central line placement & 38 & \pm 17 & 44 & \pm 11 & 6 & $16 \%$ & 0.536 \\
\hline Tunneled dialysis catheter removal & 55 & \pm 8 & 43 & \pm 8 & -12 & $-22 \%$ & 0.031 \\
\hline IVC filter placement & 33 & \pm 12 & 41 & \pm 8 & 8 & $24 \%$ & 0.200 \\
\hline Gastrostomy placement & 28 & \pm 4 & 40 & \pm 4 & 12 & $43 \%$ & 0.010 \\
\hline CT-guided lung biopsy & 43 & \pm 5 & 39 & \pm 11 & -4 & $-9 \%$ & 0.410 \\
\hline IVC filter removal & 18 & \pm 7 & 31 & \pm 8 & 13 & $72 \%$ & 0.021 \\
\hline Tunneled central line removal & 27 & \pm 12 & 27 & \pm 11 & 0 & $0 \%$ & 0.956 \\
\hline Transjugular liver biopsy & 22 & \pm 6 & 21 & \pm 4 & -1 & $--5 \%$ & 0.797 \\
\hline Chemoembolization & 25 & \pm 2 & 19 & \pm 6 & -6 & $-24 \%$ & 0.052 \\
\hline Lower extremity venous disease & 7 & \pm 2 & 14 & \pm 6 & 7 & $100 \%$ & 0.040 \\
\hline Portal hypertension management & 16 & \pm 2 & 14 & \pm 7 & -2 & $-13 \%$ & 0.370 \\
\hline Thermal ablation of HCC & 8 & \pm 3 & 13 & \pm 4 & 5 & $63 \%$ & 0.040 \\
\hline Tunneled pleural and peritoneal drainage & 12 & \pm 5 & 13 & \pm 3 & 1 & $8 \%$ & 0.670 \\
\hline Gastrostomy maintenance & 13 & \pm 4 & 13 & \pm 4 & 0 & $0 \%$ & 0.884 \\
\hline Nonlower extremity angiograms & 10 & \pm 3 & 13 & \pm 4 & 3 & 0.3 & 0.0385 \\
\hline Uterine artery embolization & 8 & \pm 4 & 7 & \pm 2 & -1 & $-13 \%$ & 0.820 \\
\hline Peripheral arterial disease & 3 & \pm 4 & 6 & \pm 3 & 3 & $100 \%$ & 0.340 \\
\hline Other & 109 & & 149 & & & & \\
\hline Total & 953 & \pm 63 & 1063 & \pm 34 & 110 & $12 \%$ & 0.01 \\
\hline
\end{tabular}

Abbreviation: HCC, hepatocellular carcinoma.

Note: Relative procedure volumes before and after the opening of the interventional radiology clinic. Values in bold are significant at the $p<0.05$ level. 
Table 2 Facility charges for selected procedures

\begin{tabular}{|l|l|l|l|}
\hline Procedure & Angiography suite & Clinic & $\begin{array}{l}\text { Annual procedures } \\
\text { completed in clinic }\end{array}$ \\
\hline Tunneled central line removal & $\$ 1875$ & $\$ 213$ & 127 \\
\hline $\begin{array}{l}\text { Implantable infusion port } \\
\text { removal }\end{array}$ & $\$ 3848$ & $\$ 213$ & 193 \\
\hline Gastrostomy change or removal & $\$ 677$ & $\$ 213$ & 26 \\
\hline
\end{tabular}

Note: Relative charges for procedures in different settings for those procedures moved to the interventional radiology clinic.

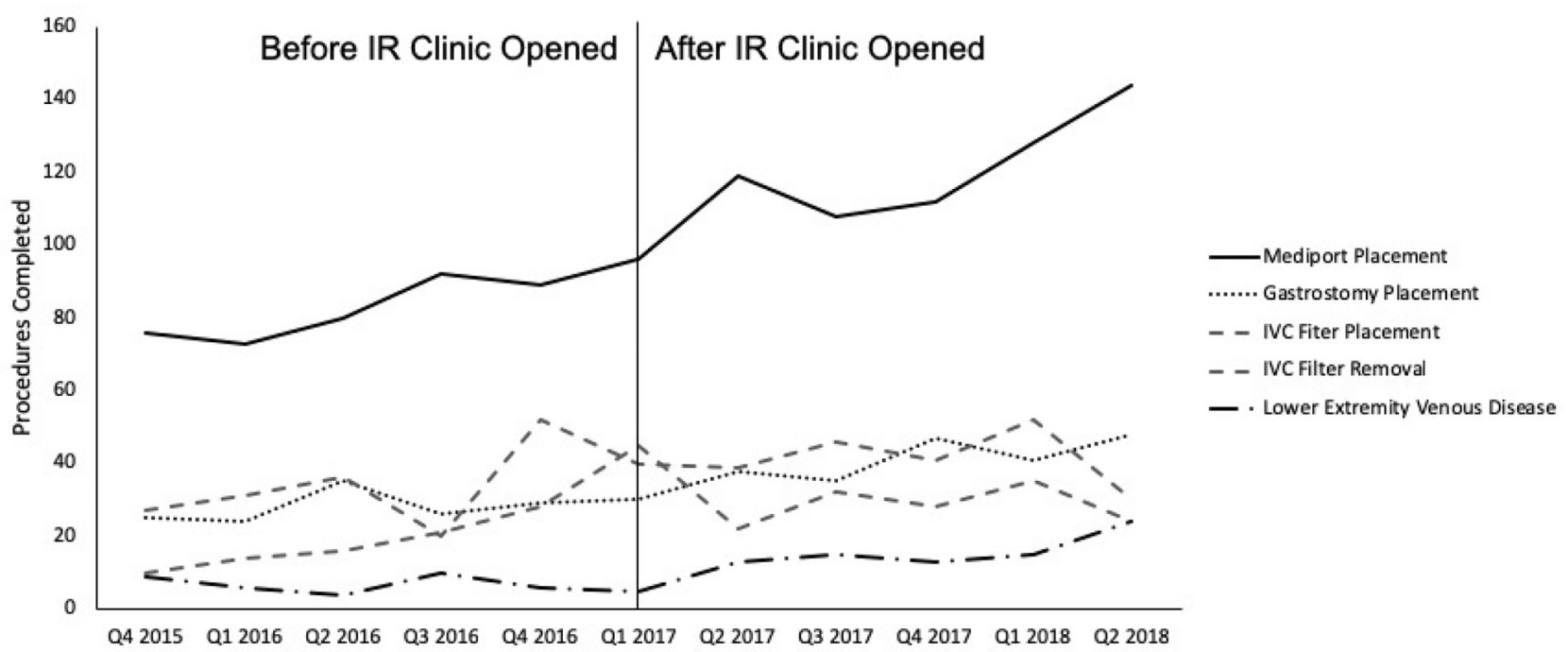

Fig. 1 The quarterly volumes of the top 5 procedures in terms of the level of increase since the opening of the clinic are shown here, with the line at 2017 showing when the clinic was opened.

procedures was due to the number of new procedures completed in the clinic (119/quarter, representing $23 \%$ of all cases performed on outpatients). As a result of completing these cases in the clinic, the angiography suite had capacity to accommodate a greater number of outpatient cases that truly required the angiography suite. The total number of cases completed in the angiography suite was unchanged (preclinic: $394 \pm 20$, postclinic: $400 \pm 26, p=0.71$ ) despite a more complex mix of cases. The number of cases completed on inpatients in the angiography suite did not significantly change after the opening of the clinic (preclinic: $542 \pm 43$, postclinic: $545 \pm 50, p=0.92$ ). After the opening of the clinic, the proportion of outpatient cases completed in the hospital decreased from $100 \%$ to $77 \%(p<0.0001)$ and the total proportion of cases completed in the hospital decreased from $100 \%$ to $89 \%$, as the remaining procedures were completed in the clinic.

\section{Impact of Clinic on Selected Case Volumes}

The volumes of several case types changed after the opening of the clinic (-Table 1). Cases that saw the largest increases were implantable infusion port placements (preclinic: $82 \pm 8$, postclinic: $118 \pm 17,44 \%$ increase, $p=0.002$ ), inferior vena cava (IVC) filter placements (preclinic: $33 \pm 12$, postclinic: $41 \pm 8,25 \%$ increase, $p=0.2$ ), IVC filter removals (preclinic: $18 \pm 7$, postclinic: $31 \pm 8,24 \%$ increase, $p=0.02$ ), treatments of lower extremity venous disease (preclinic: $7 \pm 2$, postclinic:
$14 \pm 6,100 \%$ increase, $p=0.04$ ), gastrostomy placements (preclinic: $28 \pm 4$, postclinic: $40 \pm 4,43 \%$ increase, $p=0.01$ ), and HCC thermal ablation (preclinic: $8 \pm 3$, postclinic: $13 \pm 4$, $62 \%$ increase, $p=0.04)(-$ Fig. 1 ).

Selected case types that did not significantly change in volume included uterine artery embolizations, fistulograms, portal hypertension management (defined as procedures for the creation or maintenance of transjugular intrahepatic portosystemic shunts, balloon-occluded retrograde transvenous obliteration, and Leveen shunts), tunneled dialysis catheter placement, tunneled pleural and peritoneal drainage catheter placement, and CT-guided lung biopsies.

\section{Cost Savings from Improved Management of Nephrostomies}

After the opening of the IR clinic, although the total number of nephrostomy exchange procedures increased by $16 \%$ (preclinic: $69 \pm 5.7$, postclinic: $81 \pm 7.9, p=0.03$ ), the number of inpatient exchanges was unchanged (preclinic: $25 \pm 7$, postclinic: $24 \pm 3, p=0.75$ ). This mix shift resulted in estimated annual reduction in facility charges of $\$ 468 \mathrm{~K}$.

\section{Cost Savings from Moving Procedures to the Clinic}

Procedures which could be completed in clinic witnessed a decrease in their usage of the angiography suite. Specifically, the number of implantable infusion port removals completed in the hospital decreased by $95 \%$ (preclinic: $46.8 \pm 4.1$, 
postclinic: $2.2 \pm 3.9, p<0.0001$ ), as outpatient implantable infusion port removals (average of 48/quarter) were completed in clinic. Similarly, the number of tunneled dialysis catheter removals completed in the hospital decreased by $41 \%$ (preclinic: $44 \pm 5.2$, postclinic: $26 \pm 6.5$ ), as outpatient tunneled dialysis catheter removals (average of 16/quarter) were completed in clinic. Finally, 32\% of outpatient gastrostomy maintenance procedures were completed in the clinic, resulting in no change in angiography suite usage for gastrostomy maintenance (preclinic: $16 \pm 4$, postclinic: $14 \pm 2, p=0.26$ ) despite an overall nonstatistically significant increase in the total number of gastrostomy maintenance procedures (preclinic: $39 \pm 7$, postclinic: $45 \pm 7, p=0.17$ ). As a result of moving tunneled central line, tunneled dialysis catheter, and implantable infusion port removals to the clinic setting, facility charges were reduced by $\$ 925 \mathrm{~K}$ annually for these procedures.

\section{Effects of the Clinic on Angiography Suite Usage}

Since the opening of IR clinic, the median start time of the first outpatient case was 36 minutes earlier than prior to the opening of clinic (preclinic: 9:24 am, postclinic: 8:48 am, $p<0.001$ ). The median time of completion of the last weekday, nonholiday, noncall case was 19 minutes earlier after opening the clinic (preclinic: 6:22 pm, postclinic: 6:03 pm, $p=0.004)$.

\section{Overall Clinic Key Performance Indicators}

In the last year, the clinic completed 3300 visits, reporting $\$ 700 \mathrm{~K}$ in facility revenue and $\$ 250 \mathrm{~K}$ in professional revenue against $\$ 325 \mathrm{~K}$ variable costs, which includes staff and supplies and $\$ 200 \mathrm{~K}$ in annual facility costs. As much as $35 \%$ of visits were low complexity (evaluation and management levels 1 or 2 ) and $65 \%$ were moderate or high complexity (levels 3-5).

\section{Discussion}

Opening an IR clinic at this tertiary county hospital created measurable operational and financial value. Specifically, the clinic allowed the department to increase its volumes, reduce costs for selected procedures, start more cases on time, and shorten the angiography suite workday. This clinic shows demonstrates quantitative evidence of the multifaceted value that a clinic can produce in this practice setting.

The IR clinic enabled the division to expand its number and complexity of cases. Prior to opening of the clinic, the department was capacity-constrained, partly due to use of the angiography suite for cases that could be completed in a less resource-intensive setting. By moving $10 \%$ of cases to the clinic setting (tunneled line removals, implantable infusion port removals, and gastrostomy maintenance procedures), the capacity for cases that truly required the angiography suite was effectively increased by $10 \%$, reducing facility charges for these cases by approximately $\$ 925 \mathrm{~K}$ per year. Such savings are particularly important in this tertiary, county-funded system whose inpatient resources are often at capacity, and in which the hospital has a fixed budget and has a high incidence of unreimbursed care. In particular, the ability to use the clinic to move these cases to a less resource-intensive setting allowed the IR service to accommodate significantly more implantable infusion port placements, gastrostomy placements, and IVC filter placements and removals, creating significant value for patients and health system. Previously, some of these cases had to be done by surgical services in a much less cost-effective setting, and by improving the capacity of the IR department, the IR division was able to complete a greater share of these cases.

The clinic also resulted in increased referrals for disease processes that require preprocedural evaluation and/ or follow-up. Specifically, referrals for treatment of venous disease, IVC filter removals, and thermal ablation of HCC increased along with the division's ability to provide outpatient consultation and longitudinal follow-up. Additionally, the increased space in the angiography suite due to moving procedures to the clinic allowed the division to provider faster turnaround time on outpatient procedures, which also resulted in more referrals. Previous experience has also showed increased IVC filter removal rates ${ }^{14}$ and growth in interventional oncology procedure volumes ${ }^{5}$ after the establishment of a dedicated clinic.

In addition to increased referrals, the outpatient clinic provided an opportunity for the service to reduce costs by providing preventative care to patients with nephrostomy catheters. The clinic allowed providers to deliver additional education, to ensure patients had necessary supplies, and to increase the rates of adherence to scheduled nephrostomy exchanges. As a result of this intervention, fewer emergent exchanges were needed, decreasing patient morbidity and reducing preventable facility charges by approximately $\$ 450 \mathrm{~K}$ per year. The ability of the clinic to alter the acuity of case mix has not been demonstrated before, but prior work has shown improved patient satisfaction and patient safety during IR procedures after the institution of an IR clinic. ${ }^{9}$

Utilization of the angiography suite also improved as a result of the IR clinic. Since preprocedural history and physical evaluations, as well as informed consent discussions, were completed in the clinic for outpatient procedures, less time was spent by providers on same-day workups, particularly in obtaining consent which often required the assistance of a translator. As a result, the first outpatient case started 36 minutes earlier after the opening of the IR clinic. Also, despite completing the same number of cases in the angiography suite, the last case of the day still ended 20 minutes earlier, likely because morning outpatient procedures started on time. This demonstrates the positive impact of the IR clinic on angiography suite operations and allowed faculty to end their days earlier.

This report has several limitations. Most importantly, the success of this clinic may not be generalizable to other institutions, particularly given the institution-specific goals of the clinic unique to county hospital systems. Unique institutional characteristics, including features of the clinic, patient population, and hospital system, also reduce generalizability, particularly the value of additional clinic visits for patients with 
indwelling nephrostomies. Increases in procedure volumes, such as venous interventions, could be due to broader trends supporting growth in these procedures, although the affiliated university hospital has not seen a similar trend. This report is also limited by the accuracy of the procedure names and reasons for clinic visits recorded in the electronic medical record. Additionally, opportunity costs of patients incurred by clinic visits were not captured, which may reduce the societal value of the clinic. Finally, provider time was not specifically tracked, so other initiatives could have explained the earlier completion time, although no other scheduling initiatives were tested.

\section{Conclusion}

Opening an IR clinic resulted in measurable clinical, operational, and financial benefits to the patients, hospital, and physicians in this tertiary county system.

\section{Role of Each Author}

All authors meet all four criteria for authorship as recommended by the International Committee of Medical Journal Editors (ICMJE).

- Substantial contributions to the conception or design of the work as well as the acquisition, analysis, or interpretation of data for the work.

- Drafting the work or revising it critically for important intellectual content.

- Final approval of the version to be published.

- Agreement to be accountable for all aspects of the work in ensuring that questions related to the accuracy or integrity of any part of the work are appropriately investigated and resolved.

\section{Conflicts of Interest}

None declared.

\section{Acknowledgments}

None.

\section{References}

1 Charalel RA, McGinty G, Brant-Zawadzki M, et al. Interventional radiology delivers high-value health care and is an Imaging 3.0 vanguard. J Am Coll Radiol 2015;12(5):501-506
2 Allen B Jr. Radiologists adding value through the clinical practice of interventional radiology. J Am Coll Radiol 2015; 12(4):319-320

3 Al-Kutoubi A. Admission privileges and clinical responsibilities for interventional radiologists. Cardiovasc Intervent Radiol 2015;38(2):257-260

4 Zener R, Demers V, Bilodeau A, et al. Clinical IR in Canada: the evolution of a revolution. J Vasc Interv Radiol 2018;29(4): 524-530.e2

5 Koran ME, Lipnik AJ, Baker JC, Banovac F, Omary RA, Brown DB. Procedural impact of a dedicated interventional oncology service line in a National Cancer Institute Comprehensive Cancer Center. J Am Coll Radiol 2016;13(9):1145-1150

6 Soares GM. The value of clinical interventional radiology. J Am Coll Radiol 2011;8(5):318-324

7 Abboud S, Partovi S, Nakamoto D, Azar N. The radiologist will see you now: patients' perceptions of an outpatient interventional clinic. Curr Probl Diagn Radiol 2015;46:177-180

8 Bertino R, Pai D, Beach D, et al. Establishment of an interventional radiology clinic to follow untreated abdominal aortic aneurysms. J Am Coll Radiol 2006;3(4):291-295

9 Lutjeboer J, Burgmans MC, Chung K. van Erkel ARJC. Impact on Patient Safety and Satisfaction of Implementation of an Outpatient Clinic in Interventional Radiology (IPSIPOLI-Study): a quasi-experimental prospective study. Cardiovasc Intervent Radiol 2015;38(3):543-551

10 Mouli S, Baker JC, Brown DB. Interventional oncology service development. Semin Intervent Radiol 2017;34(2):182-186

11 Siskin GP, Bagla S, Sansivero GE, Mitchell NL. The interventional radiology clinic: what you need to know. Semin Intervent Radiol 2005;22(1):39-44

12 Siskin GP, Bagla S, Sansivero GE, Mitchell NL. The interventional radiology clinic: key ingredients for success. J Vasc Interv Radiol 2004;15(7):681-688

13 McDevitt JL, Acosta-Torres S, Zhang N, et al. Long-term percutaneous nephrostomy management of malignant urinary obstruction: estimation of optimal exchange frequency and estimation of the financial impact of patient compliance. J Vasc Interv Radiol 2017;28(7):1036-1042.e8

14 Minocha J, Idakoji I, Riaz A, et al. Improving inferior vena cava filter retrieval rates: impact of a dedicated inferior vena cava filter clinic. J Vasc Interv Radiol 2010;21(12):1847-1851 